\title{
A Study of EPUB 3.0 Authoring Tools Availability in Vertical Form Function
}

\author{
Chien-Yuan Chang ${ }^{*}$, Hung-Jen Yang \\ Department of Industry Technology Education, National Kaohsiung Normal University, Kaohsiung, Chinese Taipei. \\ Email: *ccy@hcvs.kh.edu.tw, hungjen.yang@gmail.com
}

Received July $16^{\text {th }}, 2013$; revised August $15^{\text {th }}, 2013$; accepted August 22 $2^{\text {nd }}, 2013$

Copyright (C) 2013 Chien-Yuan Chang, Hung-Jen Yang. This is an open access article distributed under the Creative Commons Attribution License, which permits unrestricted use, distribution, and reproduction in any medium, provided the original work is properly cited.

\begin{abstract}
The purpose of this study was to identify the availability of authoring tools that support vertical flow function based upon empirical experiments. There were five EPUB authoring tools selected for this study. Four major editing procedures were designed for the project. The first was the support of Chinese character input. The second was vertical form editing. The third was ruby position editing, and finally, testing whether the editing software was capable of outputting a document as an e-Book in EPUB 3.0 format. Five programs were tested, and only one of the five was able to fulfill the aforementioned requirements. The usability of each program is positively verified through this study.
\end{abstract}

Keywords: e-Book; EPUB; Vertical Form; Ruby Text

\section{Introduction}

An electronic book (variously, e-Book, eBook, digital book) is a book-length publication in digital form, consisting of text, images, or both, and produced on, published through, and readable on computers or other electronic devices [1,2]. EPUB is the distribution and interchange format standard for digital publications and documents based on Web Standards. EPUB defines a means of representing, packaging and encoding structured and semantically enhanced Web content for distribution in a single-file format. EPUB allows publishers to produce and send a single digital publication file through distribution and offers consumers interoperability between software and hardware for unencrypted reflowable digital books and other publications [3].

EPUB 2 was initially standardized in 2007 as a successor format to the Open e-Book Publication Structure or "OEB", which was originally developed in 1999. A maintenance release, EPUB 2.0.1, was approved in 2010. In October, 2011, EPUB 3 was approved as a final Recommended Specification [3]. Recently the International Digital Publishing Forum (IDPF) created a technical standard "to facilitate digital content creation, distribution, and use by customers." Also known as the Open

\footnotetext{
"Corresponding author.
}

e-Book Publication Structure Container Format (OCF), the new standard allows for packaging of a digital file into a single format so that consumers with different e-Book readers will be able to share material. This will expand the availability of titles and lower the costs for publishers entering the e-Book market. For school libraries, a standard format offers an opportunity for students to exchange digital e-Books as easily as they trade paperbacks. Lower costs will also allow more schools to acquire e-books and other digital material [4].

EPUB 3.0 contains a huge array of specifications, such as multimedia specification (including MP3, H.264) the text Pronunciation engine (TTS), synchronized media integration language (SMIL), typesetting specifications (including HTML 5, CSS 3, SVG) and Asian country specific vertical text, line breaking rules, ruby position (such as pronunciation symbols marginal notes), and page rules [5].

Many East Asian scripts can be written horizontally or vertically. Chinese, Japanese and Korean scripts can be oriented in either direction, as they consist mainly of disconnected syllabic units, each occupying a square block of space [5], as shown in Figure 1.

Traditionally, Chinese, Japanese, and Korean are written vertically in columns going from top to bottom and ordered from right to left, with each new column starting 


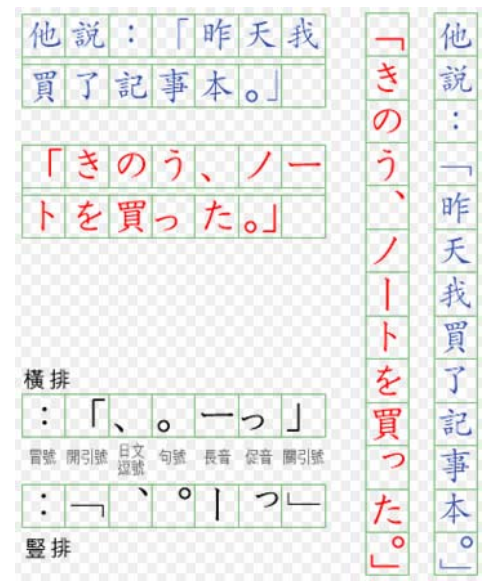

Figure 1. Japanese and Chinese vertical flow [5].

to the left of the preceding one. The stroke order and stroke direction of Chinese characters, Japanese Kana, and Korean Hangul all facilitate writing in this manner. In addition, writing in vertical columns from right to left facilitated writing with a brush in the right hand while continually unrolling the sheet of paper or scroll with the left. In modern times, it has become increasingly common for these languages to be written horizontally, from left to right, with successive rows going from top to bottom, under the influence of European languages such as English, although vertical writing is still used in Japan, Taiwan, Hong Kong and Macau frequently [5].

\section{Materials and Methods}

A EPUB 3.0 strengthens consistency and network standards to increase the performance of e-Book content in new browsers, and also EPUB 3.0 increases support for rich media content. In addition, EPUB 3.0 will also incorporate Japanese, Chinese and other languages, strengthen navigation, and add comments and other interactive features. With the EPUB 3.0 specification evolution, importing e-Book content will become livelier, and more diverse. Users can not only zoom in and out easily, set the book's font and layout, but it also supports multi-media, and more complex forms of magazines, as well as audio and video content.

Japanese scholar (Robun Birtle, 2011) indicated current trends on the standardization of the e-Book format. Apple had been already committed to the EPUB 3.0 content standard which encapsulates these requirements and Amazon is expected to at least commence EPUB support [6], so we can understand the importance of the EPB 3.0 format. According to the three articles: "How To Make An eBook" [7], "Online Vertical Layout" [8] and "An RDF-Based Platform for e-Book Publishing" [9], there were five programs that were selected to conduct this study. All five programs claimed that they could support
EPUB 3.0 format and vertical layout. The selected programs were MS-Word, MS- Publisher, Adobe In Design CS6, Sigil, and Calibre.

The purpose of this paper is to test the same four functions using each of the five programs. Four major editing procedures were designed for the experiments. The first was the support of Chinese character input. The second was vertical form editing, including the document displaying characters vertically, and arranging characters form right to left. The third was ruby position editing, and finally, testing whether the editing software was capable of outputting a document as an e-Book in EPUB 3.0 format. This paper examines the usability of the authoring tools via the aforementioned four functions.

The testing focus is on text layout because most publications in Asia, such as magazines and pamphlets, are composed of both vertical and horizontal writing. For vertical writing, the program should arrange the characters from top to bottom, and arrange lines from right to left. Another important test is for ruby text compatibility. Ruby text is special styling (shown in Figure 2,) that appears to the right of the base character or glyph. This value is provided for the special case of traditional Chinese and is used frequently in Taiwan. Ruby text (made of Bopomofo glyphs) in that context appears vertically along the right side of the base glyph, regardless of horizontal or vertical base character layout.

\subsection{Test Environment}

Hardware

- CPU: Intel Celeron $2.5 \mathrm{MHz}$

- HD: 500GB

- RAM: $2 \mathrm{G}$

- VGA: Intel G41

Operating System: MS-Windows 7

Authoring Tools Application

- Adobe In Design CS6 http://www.adobe.com

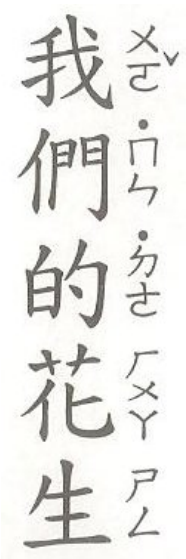

Figure 2. Ruby text appears right of the base Glyph. 
- MS-Word 2013 http://office.microsoft.com/en-us/

- MS-Publisher 2013 http://office.microsoft.com/en-us/

- Sigil 0.7.1 (freeware) https://code.google.com $/ \mathrm{p} /$ sigil/

- Calibre 0.8 (freeware) http://calibre-ebook.com/

\subsection{Steps to Create an e-Book}

Generally, there are four steps to create an e-book, listed as follows:

1) The content should be prepared in a file, such as doc/docx of Word, plain text, or html file.

2) Import prepared content file into an authoring program such as In Design, Sigil, MS-publisher, etc.

3) The imported content is edited and published into an e-Book by the authoring program.

4) Read the published e-Book via an e-reader program. 3.

The process of creating an e-Book is shown in Figure

\subsection{Test In Design CS6}

In Design is a desktop publishing software application produced by Adobe Systems. It can be used to create works such as posters, flyers, brochures, magazines, newspapers and books. We went to the Adobe website to download the trial version from URL: http://www.adobe. com, then installed the application, and launched it. We then began checking the vertical form, ruby position settings, right-to-left horizontal writing, exporting to EPUB 3.0 file format, Inputting Chinese text, etc. We created a new document, and set page size and margins. One can input his or her own text or import text from other documents.

Step 1: Checked application support for keying Chinese characters. In Design can write Chinese in the work area.

Step2: Tested word direction. Selected main menu "Object", then clicked "transform/Rotate $90^{\circ}$ ".

Step3: Tested the character right-to-left horizontal writing. Selected main menu, after forming a "Type Tool" box, then input characters into the box, selected

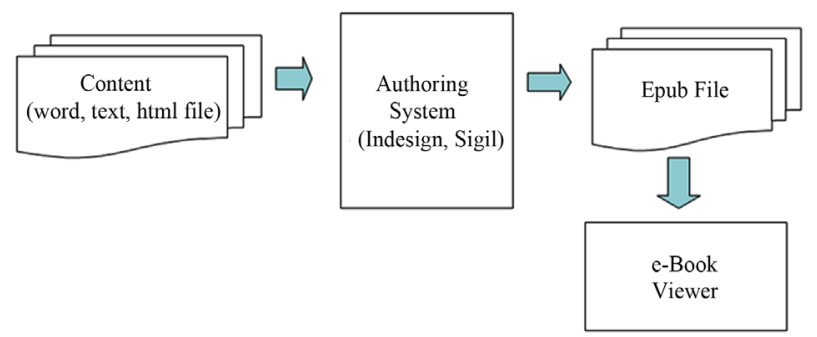

Figure 3. e-Book creation process.
"Type", then clicked "Direction/Vertical". In Design CS6 could transfer Chinese characters to vertical form, and display them from right to left and from top to bottom. The screen is shown in Figure 4.

Step4: Tested ruby function for text. We selected double text block, In Design CS6 could use Chinese fonts that place the ruby text to the right of a Chinese character. To do so, select main menu "Type", click "Font", then choose a Chinese font that includes pronunciation symbols. The screen is shown in Figure 4. Finally, save as "Other File" format, and select the EPUB 3.0 format. This screen is shown in Figure 5.

\subsection{Test MS-Word 2013}

MS Word is a word processor designed by Microsoft. It is a popular office document processing program in Taiwan. It enables the user to write articles, books, letters and documents. Download the trial version of the software from Microsoft's web site: URL: http://office.microsoft.com/ en-us/word/, then install the application. To start MS-Word, create a new Word document. Again, we want to test vertical form, text direction, and ruby positioning, and Chinese input support.

Step 1: Test support of Chinese Character entry. Chinese characters can be input into the MS-Word work area.

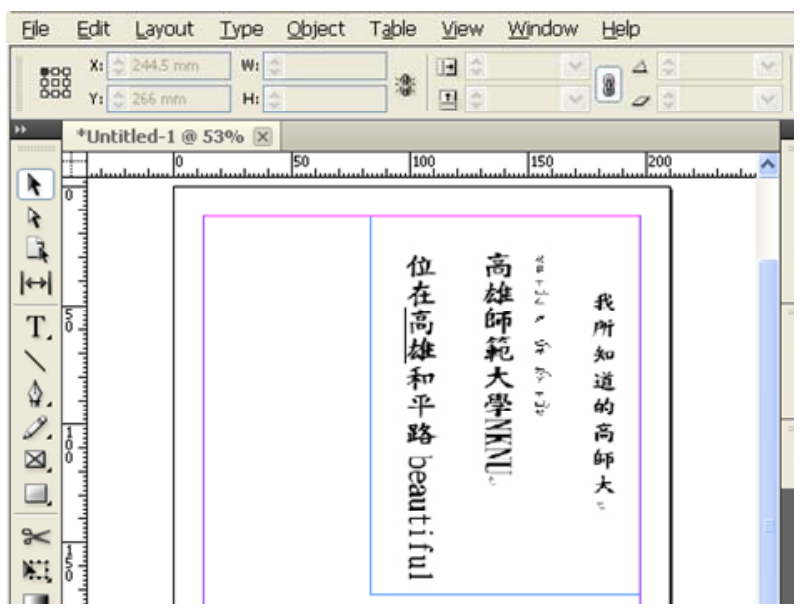

Figure 4. Vertical form.

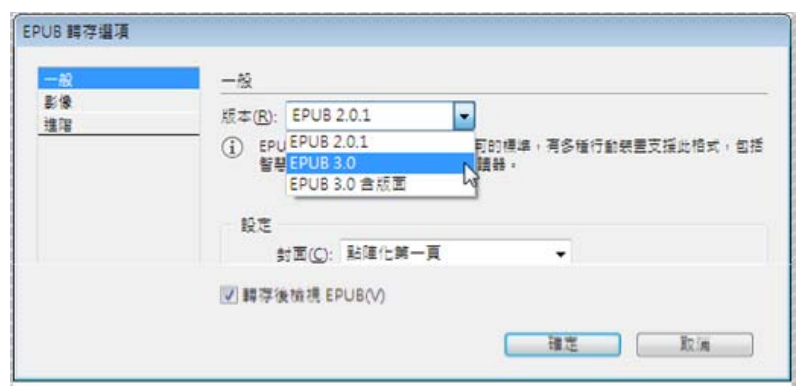

Figure 5. Saving EPUB 3.0 format e-Book. 
Step 2: Test the document direction. Select main bar "Page-Layout", using MS-Word one can select "Character Direction" or "Page Direction" to transform characters.

Step 3: Test character Right-to-left horizontal and topto-down writing. Select main menu "Page-Layout", then click "Character/Vertical form", MS-Word could accomplish this task. The screen is shown in Figure 6.

Step 4: Test ruby function for text. Select main bar "Start", click toolbar "Phonetic" to set relevant parameters then click "OK". MS-Word could save our file in docx, PDF, and html formats, afterward, MS-Word could use other programs to convert the files into whatever other e-Book formats you desired. However, MS-Word itself could not save as the new specifications of EPUB 3.0.

\subsection{Test MS-Publisher 2013}

Microsoft Publisher is an entry-level desktop publishing application from Microsoft, differing from Microsoft Word in that the emphasis is placed on page layout and design rather than text composition and proofing. Download the try version software from URL: http:// office. microsoft.com/en-us/, then install the application. We could choose to begin a new publication based on an installed or online template or create our own design on a blank page.

Step 1: Testing support for Chinese Characters entry. MS-Publisher could accept Chinese characters in the title.

Step 2: Test the document direction. Select main Bar "Page-Layout", in MS-Publisher one can select "Character Direction" or "Page Direction" to transform the characters.

Step 3: Test Right-to-left horizontal and top-to-down

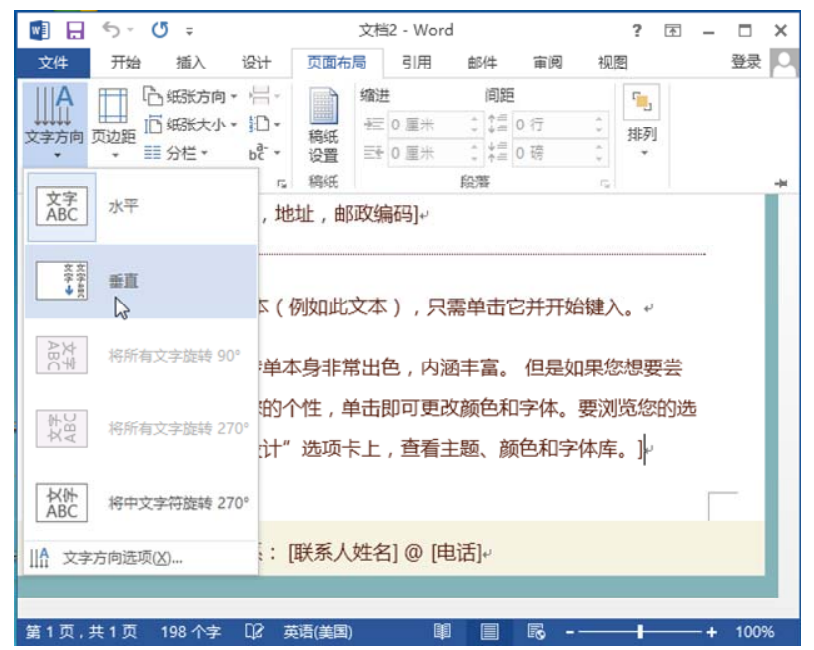

Figure 6. Changing the direction of Chinese text in a paragraph. writing. Select "Page-Layout" from the main menu, then click "Character/Vertical Form", MS-Publisher could accomplish this task. The screen is shown in Figure 7.

Step 4: Test ruby function for text. Select "Start" from the main bar then click toolbar "Notation", to set relevant parameters, and then click "OK". MS-Publisher could save our file in PUB, PDF and HTML document formats. MS-Publisher could also save in other picture formats, such as PNG, JPG, GIF, and BMP image file format, too. However, MS-Publisher could not save as the new specifications of EPUB 3.0.

\subsection{Test Sigil}

Sigil is an open-source editor for EPUB e-Books developed by Strahinja Marković in 2009 and has been maintained by John Schember since 2011[10]. Download the software from URL: https://code.google.com/p/sigil/, then install the application, and launch it. Sigil can create a new book by selecting "File" from the main menu, then click "New" to create a new blank book. We wanted to test vertical form, text direction, and ruby position, and Chinese character input support.

Step1: Testing support of Chinese character entry. Sigil could type and edit Chinese characters.

Step2: Test text direction. We could only type using horizontal form. It is no transform character function.

Step3: Test character Right-to-left input. Sigil has no vertical form function in its main menu. It just has left-to-right writing.

Step4: Test ruby function for text. It could not add text to the right side of the base character. The screen is shown in Figure 8.

This application could also open and edit EPUB books, instead of just converting them from other formats to EPUB. Since version 0.7, Sigil supports embedded video or audio in EPUB.

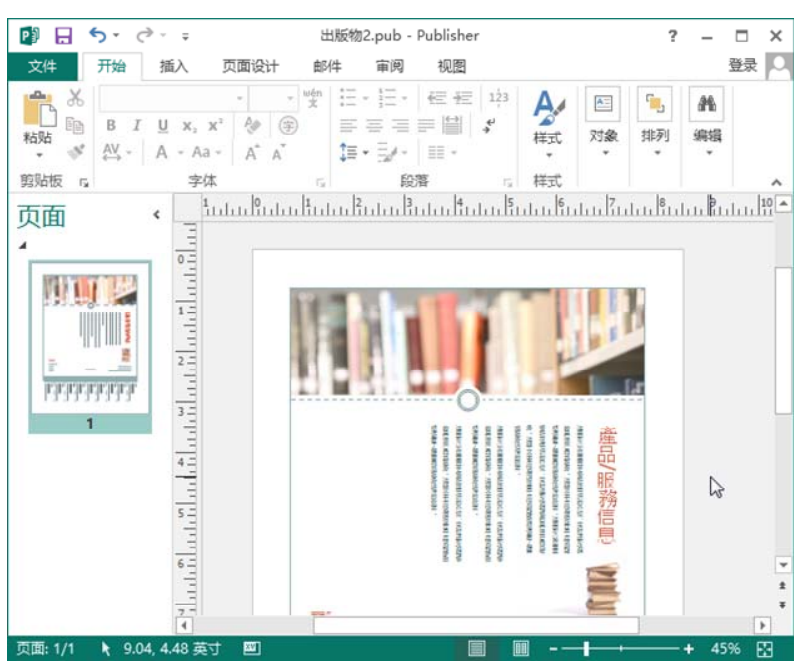

Figure 7. Rotate the direction of Chinese text. 


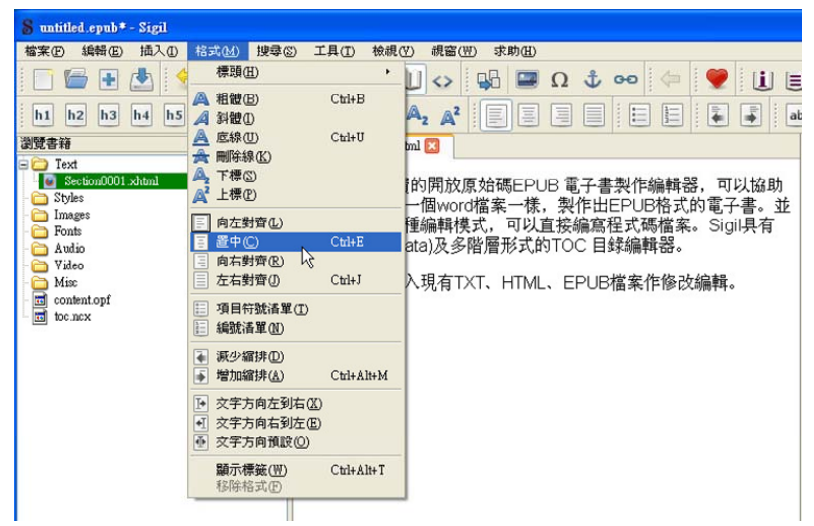

Figure 8. Rotate the direction of Chinese text.

\subsection{Test Calibre}

Calibre is a free and open source e-Book library management application developed by users of e-Books for users of e-Books. It could view, convert and catalog e-Books in most of the major e-Book formats [11].

Download the software from URL: http://calibreebook.com/. When first running Calibre, the Welcome Wizard starts and will set up Calibre for your e-reader device. Conversion is easy for Calibre. Just select the book you want to convert then click the "Convert Books" button. Ignore all the options for now and click "OK". The little icon in the bottom right corner will start spinning. Once it's finished spinning, your converted book is ready. Click the "View" button to read the book.

\section{Result and Discusion}

This is an open standard adopted by Apple (iOS), Barnes $\&$ Noble (Nook) and many other makers of e-Book readers (such as Sony). Thankfully, Amazon has said that its next Kindle will also support EPUB 3.0 [7]. EPUB 3.0 files are commonly used for electronic documents over various platforms due to the advanced style control and the anticipated future growth in popularity of the free reading software.

With the emergence of the Tablet PC and smart phones, digital reading has gradually become a part of many people's lives, and e-Books are an important contribution. However, we think that e-Books should be combined with local culture and there are many publications that use vertical form in Taiwan. Especially, elementary schools need to use ruby text for phonetic alphabet symbols for textbooks in Taiwan.

In the printed world, vertical script order still sells. In China, where the horizontal order is now the norm, occasionally there are major publications employing traditional text layout. The monumental series Zhonghua Zai Zao Shan Ben Cong Shu (Re-making of the rare books of China series) is one example. In Japan, school textbooks of the national language still employ vertical text from primary level one through senior high levels. The message is clear: let's not forget our origins and keep tradition alive. Library workers, as facilitators of information transmission and knowledge preservation regardless of the medium, may also contribute. The World Wide Web is open to all languages and in whatever manifestation [8].

Today, the left-to-right direction is dominant in all various languages for horizontal writing: this is due partly to the influence of English, and partly to the increased use of computerized typesetting and word processing software, most of which does not directly support the right-to-left layout of East Asian languages. Most software can not support Chinese vertical form and ruby function. The ruby is an assistant function for readers used to annotate a base character. Ruby text displays a pronunciation guide for characters that are likely to be unfamiliar to the reader.

This paper has examined five sets of available software that could be used to make a vertical form eBook. We understand their different functions in supporting the EPUB 3.0 format. The test resulted are show in Table 1.

According to the above test results. MS-Word and MS-Publisher were both good text editors, and it was easy to key-in Chinese characters, both could also display characters verticals. MS-Word files could be saved in pdf file format. They belong to Microsoft company design software, they are just general document processing tools, but not professional e-Book making tools. Saving files in EPUB 3.0 format was unavailable.

These results show that only InDesign supports Chinese vertical form and supports EPUB 3.0 format. In this study, the MS-publisher 2013 could directly save files as the old EPUB format, but it does not support the new EPUB 3.0 format. Calibre boasts powerful functions and it could convert different brands and formats of e-Books. Calibre's conversions were incredibly fast. It basically converts your text rapidly, from any format, to any format. If you try to send a book to your e-reader, and the

Table 1. Software comparison.

\begin{tabular}{ccccc} 
Function & $\begin{array}{c}\text { Support } \\
\text { Chinese } \\
\text { Available }\end{array}$ & $\begin{array}{c}\text { Vertical } \\
\text { Form } \\
\text { Available }\end{array}$ & $\begin{array}{c}\text { Ruby } \\
\text { Position } \\
\text { Available }\end{array}$ & $\begin{array}{c}\text { EPUB 3.0 } \\
\text { Format } \\
\text { Available }\end{array}$ \\
\hline InDesign CS6 & yes & yes & yes & yes \\
MS-Word & yes & yes & yes & no \\
MS-Publisher & yes & yes & yes & no \\
Sigil 0.7.1 & yes & no & no & no \\
Calibre 0.9.26 & yes & no & no & no \\
\hline
\end{tabular}


book is in the wrong format, Calibre simply converts it in the transfer process. However, it is not a good editing tool. Sigil is an EPUB two format text editor and does not currently support all the features of the EPUB 3.0 format. But it does support audio and video files which are the most often requested parts of EPUB 3.0, and does allow you to preserve EPUB 3.0 tags.

The usability of each program was positively verified through this study. The study examined each application's ability to support the EPUB 3.0 format, support typing from right to left, top to bottom notation, which corresponds to the Chinese, Japanese and some other traditional Asian text formats. So with these EPUB 3.0 features corresponding to Chinese and Japanese readers' reading habits, the development of e-books will become more popular in Asian countries. If any publisher wants to publish an e-Book in Chinese, this study recommends using the EPUB version for e-Books in the authoring application. Despite the increasing popularity of e-Books and e-readers, traditional Chinese publications still have yet to fully adopt the EPUB format to achieve universal standardization. There are a lot of e-readers that support EPUB, including the iPad, Sony Reader, Barnes \& Noble Nook, Ibis Reader, and Stanza [12].

\section{Conclusions}

The transformation of e-Books and digital publishing presents challenging technical specifications to unify. The e-Book is a fast-growing technological market. It needs a standard e-reader to increase the popularity and the interaction between the author's content and readers. The EPUB 3.0 authoring tool promotes the development of e-Books and is also forcing the publishing industry into a new phase. This standard could be a new foundation for the market. It could be implemented for mass market acceptance. Some e-Book publishers are eager to gain a market share. In addition, each manufacturer seems to have accepted the standard format e-Book e-reader for mobile devices in an existing EPUB 3.0 system. Publishers are facing the challenge of updates to technologies.

The aim of this paper was to explore whether the EPUB 3.0 editing software could provide the functions of editing vertical text, from right to left and from top to bottom. It is a very important and traditional way to display text in languages such as Chinese and Japanese. Early computer installations were designed only to support left-to-right horizontal writing based on the United States. Today, most computer programs do not fully support the vertical writing system. Few programs on the market support Chinese and vertical editing.

A large variety of eBook's contents and titles are still not available due to the lack of a standard format. Content providers are still using many proprietary formats for each of the reading devices and software [13]. However, most advanced word processing and publication software which targets the East Asian region support the vertical writing system either fully or to a limited extent. Most e-Book editors do not currently support all features of EPUB 3, nor do they support vertical form Chinese. I found that of the five programs in my study, the most powerful available authoring tool in vertical form function was In Design. Only In Design CS6 could edit Chinese vertical display and In Design CS6 also supports CSS styles that enable both horizontal and vertical layout and both left-to-right and right-to-left writing. EPUB 3.0 e-Book format is currently incompatible with some versions of e-readers. We think the perfect vertical form editor application could be published in future versions.

Up until now EPUB 3.0 editing tools are properly authenticated before publication. The market doesn't offer any program that can fully support Chinese vertical and right-to-left input. Therefore, this study significantly affects the digital publishing industry which affects e-Book Readers and publishers. With a unified e-Book format, the e-Book could spread on the internet, reduce production costs, and greatly benefit consumers. On the other hand, the article finally found very few programs using the EPUB e-Book standard to promote, so for now there is a small number of e-Book formats. Of course, there are also some commercial e-Book platforms that can be downloaded for free that use EPUB like Sigil, and Calibre. Also other e-book platforms can download some free public versions of the book EPUB files. Currently one still needs to pay for the best software, In Design. In Design has powerful features and functions in EPUB 3.0 format.

Application versions of the e-Book update fast, and this study suggests that software developers should consider the Chinese vertical format. Programmer's designs could support EPUB 3.0 format and be open source software that could support cross-platform. It should support both WYSIWYG and code-based editing of EPUB files, and should include multimedia and hypertext links in e-Book. For example, an e-Book that can allow the reader to view video clips, listen to sound and narrations, or jump to a location on the Web simply by selecting a link. Accessibility features for e-Books may also be implemented, such as text-to-speech or Braille output programs that could increase access for the blind.

\section{REFERENCES}

[1] Wikipedia, "E-book," 2013. http://en.wikipedia.org/wiki/E-book

[2] W.-S. Sohn, S.-K. Ko, K.-H. Lee, S.-H. Kim, S.-B. Lim and Y.-C. Choy, "Standardization of eBook Documents in the Korean Industry," Computer Standards \& Inter- 
faces, Vol. 24, No. 1, 2002, pp. 45-60. doi:10.1016/S0920-5489(01)00103-9

[3] International Digital Publishing Forum, 2013. http://idpf.org/epub

[4] Shonda Brisco, "E-books Get a Rewrite," School Library Journal, Vol. 53, No. 7, 2007, p. 24.

[5] Wikipedia, "Horizontal and Vertical Writing in East Asian Scripts," 2013. http://en.wikipedia.org/wiki/Horizontal_and_vertical_writ ing_in_East_Asian_scripts

[6] R. Birtle, "The Development and Future of the Japanese Ebook Market," Publishing Research Quarterly, Vol. 27, No. 4, 2011, pp. 345-353. doi:10.1007/s12109-011-9247-y

[7] Smashing Magazine, "How to Make an eBook," 2013. http://www.smashingmagazine.com/2011/09/29/how-tomake-an-ebook/
[8] L. Chor, "Online Vertical Layout," 2013. http://www3.telus.net/public/lchor/ovltext2011.htm

[9] K. Dittawit and V. Wuwongse, "An RDF-Based Platform for E-Book Publishing," Lecture Notes in Computer Science, Vol. 7008, 2011, pp. 267-276. http://link.springer.com/chapter/10.1007/978-3-642-2482 6-9_33

[10] Sigil 0.7.1, 2013. https://code.google.com/p/sigil/

[11] Calibre, 2013. http://calibre-ebook.com/

[12] The Book Designer, "Ebooks \& Ebook Readers," 2013. http://www.thebookdesigner.com/ebooks-ebook-readers/

[13] K.-H. Lee, G. Nicholas and M. Victor, "Standardization Aspects of eBook Content Formats," Computer Standards \& Interfaces, Vol. 24, No. 3, 2002, pp. 227-239. doi:10.1016/S0920-5489(02)00032-6 